\title{
2D Auxetic Metamaterials with Tuneable Micro-/Nanoscale Apertures
}

\author{
Luke Mizzi $^{1 *}$, Enrico Salvati ${ }^{2,3 *}$, Andrea Spaggiari ${ }^{1}$, Jin-Chong Tan ${ }^{4}$, Alexander M. Korsunsky ${ }^{2 *}$ \\ ${ }^{1}$ Department of Engineering Sciences and Methods, University of Modena and Reggio Emilia, Reggio Emilia, \\ Italy \\ ${ }^{2}$ MBLEM, Department of Engineering Science, University of Oxford, OX1 3PJ Oxford, United Kingdom \\ ${ }^{3}$ Polytechnic Department of Engineering and Architecture (DPIA), University of Udine, Via delle Scienze 208, \\ Udine, 33100, Italy \\ ${ }^{4}$ Multifunctional Materials \& Composites (MMC) Laboratory, Department of Engineering Science, University of \\ Oxford, OX1 3PJ Oxford, United Kingdom \\ *corresponding authors emails: Luke Mizzi luke.mizzi@unimore.it, Enrico Salvati enrico.salvati@uniud.it and \\ Alexander M. Korsunsky alexander.korsunsky@eng.ox.ac.uk
}

\begin{abstract}
Modern advanced manufacturing technologies have made possible the tailored design and fabrication of complex nanoscale architectures with anomalous and enhanced properties, including mechanical and optical metamaterials; structured materials which are able to exhibit unusual mechanical and optical properties that are derived from their geometry rather than their intrinsic material properties. In this work, we fabricated for the first time an ultrathin 2D auxetic metamaterial with nanoscale geometric features specifically designed to deform in-plane by using focused-ion-beam milling to introduce patterned nano-slits within a thin membrane. The system was mechanically loaded in-situ and exhibited in-plane dominated deformation up to $5 \%$ tensile strain and a Poisson's ratio of -0.78 . Furthermore, the porosity and aperture shape of the metamaterial have been shown to change considerably upon the application of strain, with pore dimensions showing a fourfold increase at 5\% strain. This mechanically-controlled tuneability makes this metamaterial system an ideal candidate for use as a reconfigurable nanofilter or a nano light-modulator.
\end{abstract}

\section{Introduction}

In recent years, ground-breaking advances in the field of rapid prototyping have contributed to the realization of micro- and nano-architectured materials which were previously unachievable using traditional macro-scale manufacturing processes. Depending on the geometric complexity, dimensionality, scale, resolution and material requirements of the micro- 
/nanostructure in question, a variety of advanced fabrication techniques may be used nowadays to produce small scale architecture systems [1,2]. These include a wide range of stereolithography methods [3-6], droplet deposition systems [7], self-assembly macromolecules [8,9] and vat photopolymerization [10,11] techniques amongst others. Nanoscale systems produced using these methods have been shown to possess a wide spectrum of remarkable characteristics such as plasmonic properties $[12,13]$, piezoelectric capabilities [14] and mechanically-derived anti-microbial activity [15]. Focused ion-beam lithography is one method which particularly shows a great deal of promise for the design of patterned smallscale architectures. In this technique, the substrate is bombarded with heavy ions, typically $\mathrm{Ga}^{+}$, resulting in surface modifications. Depending on the resistance and ion speed, the substrate is sputtered, milled or implanted with the colliding ions. Due to the high precision and resolution of this method, FIB is extremely well suited towards the design of architectures with nano-scale geometric parameters and is currently widely used for micro-machining of nanotools [16-18] as well as for advanced 3D microstructural analysis of materials including depth and cross-section profiling [19-21].

This technique has also been employed to produce plasmonic metamaterials [22-24] where by exploiting nano apertures or etches produced using FIB milling, one may alter the refractive index of the surface material. Thus far, a number of geometries have been proposed, both 2D and 3D, using a variety of patterns, ranging from etched structures [25], complex periodic hole networks [26,27], spiral chiral structures [28-31] and truss networks [32]. The majority of patterned geometries produced thus far using FIB milling are designed primarily for rigid-body situations, i.e. they are not implemented for applications where the metamaterial surface or structure is expected to undergo significant deformation. This, in turn, limits the versatility and tuneability of these structures since once the perforated and/or etched architecture of the system is established following manufacture, the overall porosity and aperture shapes of the metamaterial cannot be changed without re-modifying the geometry using similar or other nanofabrication methods. In view of this, the advantages of having a deformable micro/nanostructure are undeniable since a system with reconfigurable aperture sizes and shapes could in turn possess tuneable optical properties through the application of an external stimuli such as mechanical loads [33] or electrically-induced actuation [12,34].

Two-dimensional auxetic [35-37] metamaterials present a particularly ideal fit for this purpose. Many of these systems, which possess a negative Poisson's ratio as a result of their structure rather than intrinsic material properties, are porous and, furthermore, the planar void area and 
pore shape of these systems may be easily controlled through the application of mechanical loading. Deformation mechanisms leading to auxetic behaviour are also scale-independent, meaning that a mechanism which is active at the macroscale should be reproducible at lower levels such as the micro- and the nanoscale. A large spectrum of auxetic mechanisms exist in literature with diverse deformation modes including chiral [38,39], rotating rigid unit [40,41], re-entrant [42,43] and sliding mechanism [44] systems. However, reproducing these geometries with nanoscale lattice parameters presents a number of difficult challenges from both a design and fabrication viewpoint. For example, residual stress effects during FIB-milling make the precise fabrication of structures with thin, slender ligaments extremely difficult $[31,45]$. Another factor is that in order for a $2 \mathrm{D}$ auxetic system to deform in a predominantly in-plane manner and hence exploit its full auxetic potential, the depth dimension of the system must be of sufficient thickness to avoid out-of-plane deformations. Since FIBmilling can only be used to accurately perforate very thin films, this means that the lattice parameters of the resultant mechanical metamaterial must be of similar or smaller dimensions in order to obtain a system which deforms solely in-plane. These problems were observed by Valente et al. [33] who used FIB-milling to produce various micro- and nanoarchitectures based on classic re-entrant honeycombs where due to design limitations the auxetic potential of these systems was significantly reduced owing to these factors.

In this work, we manufactured a planar auxetic metamaterial (shown in Figure 1) using FIBmilling which overcomes these problems. The metamaterial deforms in-plane and exhibits a negative Poisson's ratio of -0.78 over a $5 \%$ strain range. The system was produced through the introduction of alternating slit perforations [46-50] arranged in a perpendicular manner with respect to one another, resulting in a system which deforms via the rotating squares [40] mechanism.

\section{Methodology}

A Tescan LYRA3 GM Ga+ FIB machine was used to produce patterned slit perforations with a width, $w$, of $150 \mathrm{~nm}$ on a $360 \mathrm{~nm}$ gold-plated silicon nitride membrane ( $200 \mathrm{~nm} \mathrm{Si} 3 \mathrm{~N}_{4}$ covered with $150 \mathrm{~nm}$ Au plating separated by a $10 \mathrm{~nm} \mathrm{Cr}$ adhesion layer). The slit perforations were designed with a length, $p$, of $8 \mu \mathrm{m}$ and a separation distance, $s$, of $300 \mathrm{~nm}$ and the resultant square repeating unit has a length of $c a .8 .9 \mu \mathrm{m}$ (see Figure 1b). A total of 5 repeating units were milled in the $x$-direction, while 7 repeating units were replicated in the $y$-direction. To 
minimise the interaction of the pristine membrane with the manufactured metamaterial, material removal was performed at the sides and at the top of the structure, as shown in Figure 1a. Additionally, with the aim of minimising out-of-plane deformations before and during the stretching test, a connection with the surrounding membrane was preserved at the top side. Care was taken to reduce concentrations of stress by rounding any sharp corner, sites of potential crack initiation and therefore origin of premature failures. The FIB manufacturing process of the sample slits and contouring was performed using $30 \mathrm{keV}$ of energy, at a current of $0.3 \mathrm{nA}$ and ion dose of $1.0 \times 10^{9}$ ions $/ \mathrm{mm}^{2}$. Using these FIB process parameters, the final sample shape was obtained from the pristine material in about 2 hours.

This configuration results in a self-mounted and supported sample for the execution of a classic tensile test, but at the micro-scale. The sample was loaded in displacement mode by means of a nanomanipulator. This tool was manually inserted inside a custom-sized round perforation (cut using the FIB) at the top part of the sample and 'welded' together by a controlled deposition of platinum through a Gas Injection System (GIS) embedded in the FIB-SEM microscope, as illustrated in Figure 1a. The system was subjected to tensile strains by small stepwise actuation using the nanomanipulator. At each step, the nanomanipulator was moved in the $y$-direction by a small distance, and, while the nanomanipulator was fixed in position, a high-resolution image was captured using the SEM before moving on to the next actuation step. To evaluate the Poisson's ratio of the system, the axial and transverse deformation of the central metamaterial repeating unit was evaluated using post-processing image analysis tools in Matlab (see Supplementary Information).

a)

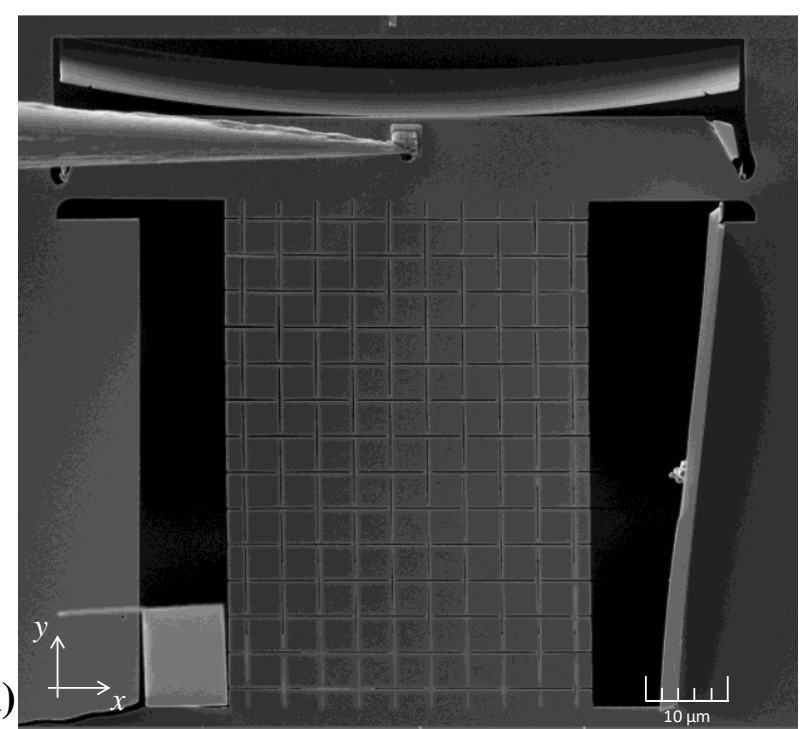

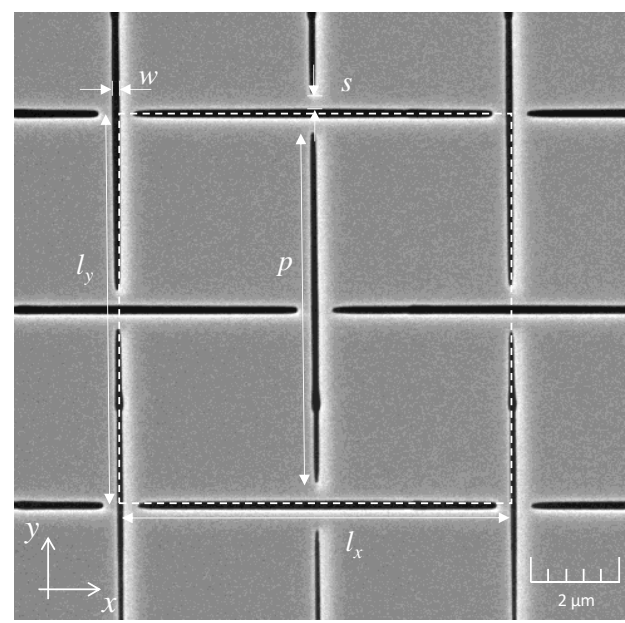

b) 
Figure 1: SEM images of a) full metamaterial geometry and b) a zoomed in repeating unit (indicated by dashed lines) from a unit cell near the centre of system before the application of tensile strain.

\section{Results and Discussion}

The system was found to possess a Poisson's ratio of approximately -0.78 for tensile strains ranging from 0 to 5\% (see Figure $\mathbf{2 b}$ ). The Poisson's ratio was also revealed to be more or less constant over this strain range. As one may observe from Figure 2a, where the deformation of one of the central repeating unit is shown, the in-plane rotation of the square units is plainly visible and there is no marked 'shadowing' indicating that components of the system are going out-of-plane. This deformation mode is characteristic of the in-plane deformation of the rotating squares mechanism and shows that despite the thinness of the membrane $(d=360 \mathrm{~nm})$ and the fact that the slit separation parameter, $s$, has a comparable magnitude, this mechanism is functional over this strain range. In order to confirm this observation, additional mechanical tests were conducted on macro-scale systems corresponding to the FIB-milled metamaterial. These systems were made from rubber and Kapton and were fabricated using direct laser cutting by employing a Universal ${ }^{\circledR}$ Laser Cutter. The slits were set to $c a .300 \mu \mathrm{m}$ thick and the systems were loaded using a Deben ${ }^{\circledR}$ microtensile loading devices as shown in Figure 2f,g. Further details on the manufacturing process of these systems may be found in the Supplementary Information section. The rubber and Kapton systems represent two extremes of the joint region thickness, $s$, to out-of-plane thickness, $d$, ratio of system. While both systems were designed with an $s$ value of $400 \mu \mathrm{m}$, the out-of-plane thickness of the rubber sheet was $1000 \mu \mathrm{m}$ whereas that of the Kapton sheet was $75 \mu \mathrm{m}$ resulting in $d / s$ ratios of 2.5 and 0.188 respectively. In addition, a nonlinear 2D Finite Element (FE) simulation of a system identical to the FIB-milled metamaterial under plane-stress conditions was conducted as well (see Supplementary Information). 
Silicon Nitride Metamaterial (FIB-Manufactured)

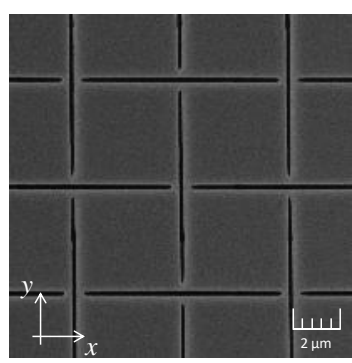

a)

$\varepsilon_{y}=0$

Finite Element Simulation

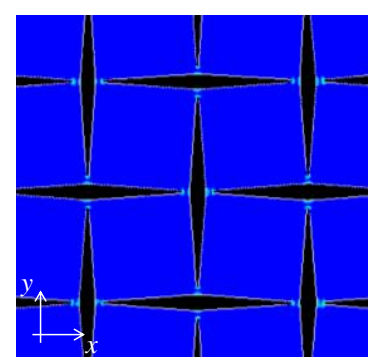

c)

$\varepsilon_{y}=0.050$

Rubber Metamaterial

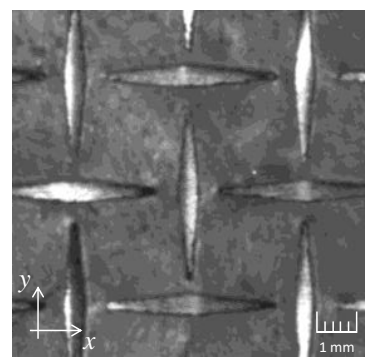

e)

$\varepsilon_{y}=0.049$

d)

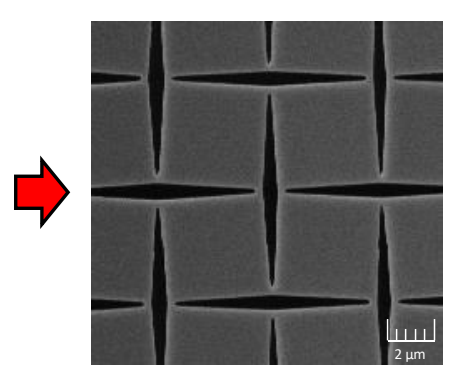

$\varepsilon_{y}=0.052$

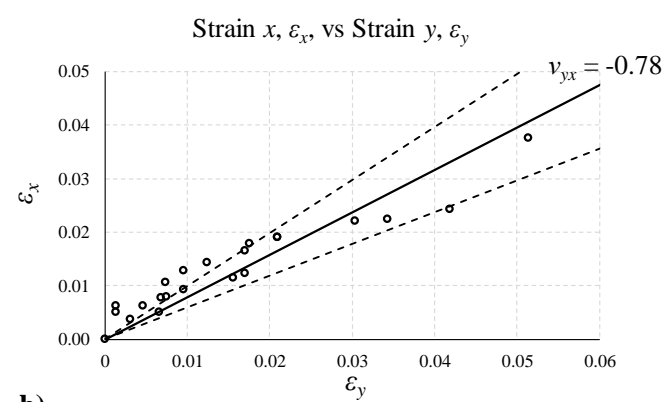

b)

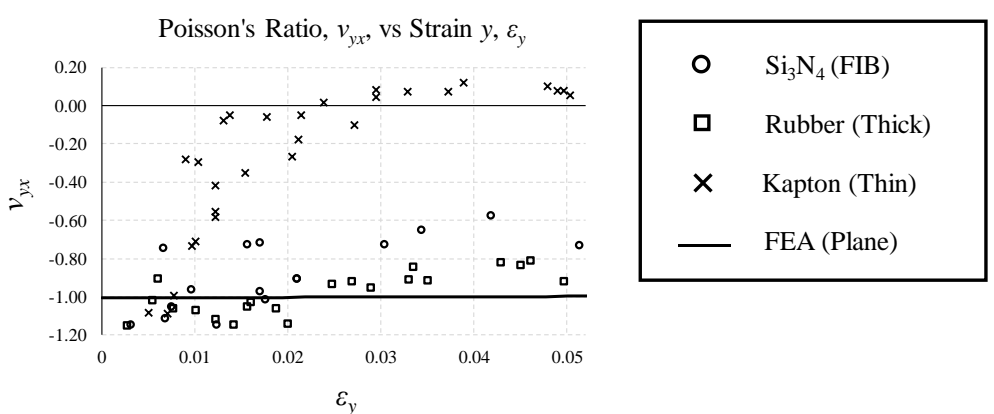

Rubber Metamaterial mounted on Microtensile loader

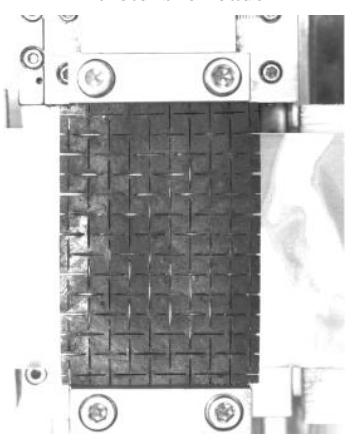

Kapton Metamaterial mounted on Microtensile loader

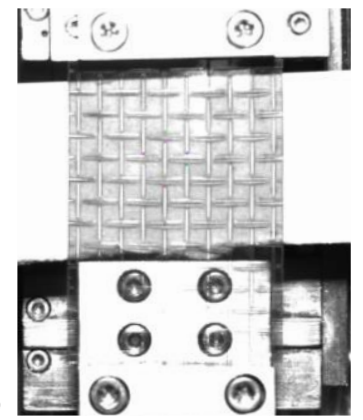

Figure 2: a) SEM image of a central repeating unit of the silicon nitride FIB-milled system before and after deformation and b) linear regression of strain-strain graph for the same system considering measurement errors (dashed lines indicate $95 \%$ confidence interval values using the $\mathrm{Chi}^{2}$ test). Central repeating unit of c) the Finite Element simulation and e) the rubber macroscale system at 5\% strain. d) Poisson's ratio vs engineering strain graphs for the silicon nitride, Kapton, rubber and FE simulation (error bars are provided in the Supplementary Information). Images of the $f$ ) rubber and g) Kapton perforated metamaterial sheets mounted on the microtensile loader before the application of tensile strain.

As evident from Figure 2d, the Poisson's ratio values of the macroscale rubber metamaterial and the micro-/nanoscale FIB-milled metamaterial are extremely similar. Both systems also show values which are in very good agreement, albeit slightly lower in magnitude, in comparison to the FE simulation, which, as expected, shows a constant Poisson's ratio of -1 over $5 \%$ strain. The deformed shape of all three systems at 5\% strain are also almost completely identical as shown in Figures 2a,c,e. On the other hand, the Poisson's ratio of the Kapton system, which has a very low out-of-plane to 'joint' region thickness ratio, varies significantly with respect to the other three systems. In fact, the Poisson's ratio of this system is not constant 
throughout and the system exhibits diminutive auxetic behaviour at strains lower than $2 \%$ before switching to a positive Poisson's ratio. This behaviour arises due to the fact that rather than rotating with respect to one another, the square units bend and stretch out-of-plane in a wave-like manner. This behaviour has previously been observed and reported in macroscale rotating square systems which are either extremely thin or in systems where the rotating mechanism has reached its fully-opened conformation [51]. Besides the loss of auxeticity, this type of deformation also results in the formation of irregularly-shaped apertures upon loading which are not directly oriented with the main plane in which the system is loaded. This is in complete contrast to that observed for the systems which deform predominantly in-plane. For these systems, the apertures take the shape of rhombic pores when the system is loaded, with the geometric dimensions of the pores being directly controlled by the extent of applied strain. As mentioned previously, the extent of mechanically controllable porosity and void shape is a crucial factor in determining the potential of this system for use as a reconfigurable optical metamaterial. In Figure 3, we quantify this variability in pore size of the FIB-milled metamaterial system in terms of the maximum rhombic pore dimension, $p_{\max }$, the percentage void area, $\% A_{V}$, and the maximum diameter of a spherical body, $d_{s}$, which can pass through the pore at a given strain for the central repeating unit of the system. These parameters were measured and/or calculated using image analysis tools in Matlab. The surface void percentage area, $\% A_{V}$, is defined as the total surface void area of a repeating unit over total overall repeating unit area percentage and $p_{\max }$ as the $p_{2}$ dimension of the pore (see Figure 3a). The latter dimension, along with the other pore parameter $p_{1}$, was used to calculate the maximum permissible diameter of a passable spherical body, $d_{s}$, using the following equation:

$$
d_{s}=\sqrt{\left(\frac{p_{1}}{\left.\frac{p_{2}}{p_{1}-w}+\frac{p_{1}-w}{p_{2}}\right)^{2}+\left[\left(\frac{p_{1}}{\frac{p_{2}}{p_{1}-w}+\frac{p_{1}-w}{p_{2}}}\right) \frac{p_{2}}{p_{1}-w}\right]^{2}}\right.}
$$

This equation takes into account the fact that the pore has a rectangular shape at $\varepsilon_{y}=0$ with non-zero porosity and that a minimum pore thickness of $w$ is retained at all times during deformation at the two corner regions of the pore defining the $p_{2}$ parameter. Further information on the pore analysis methods is presented in the Supplementary Information. 
a)
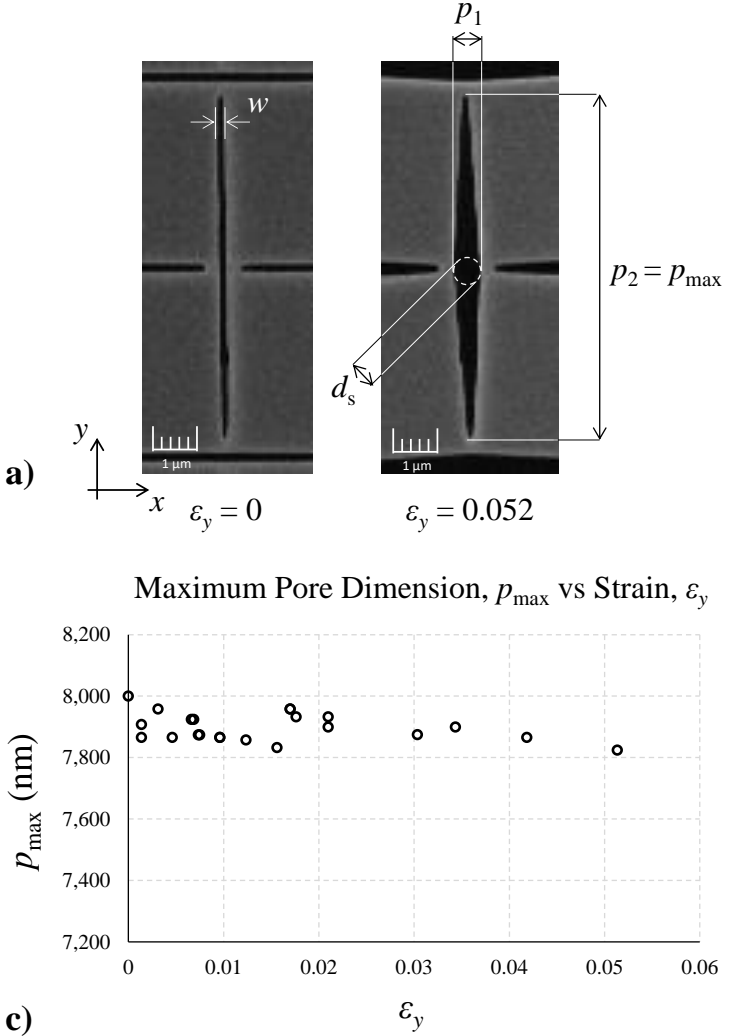
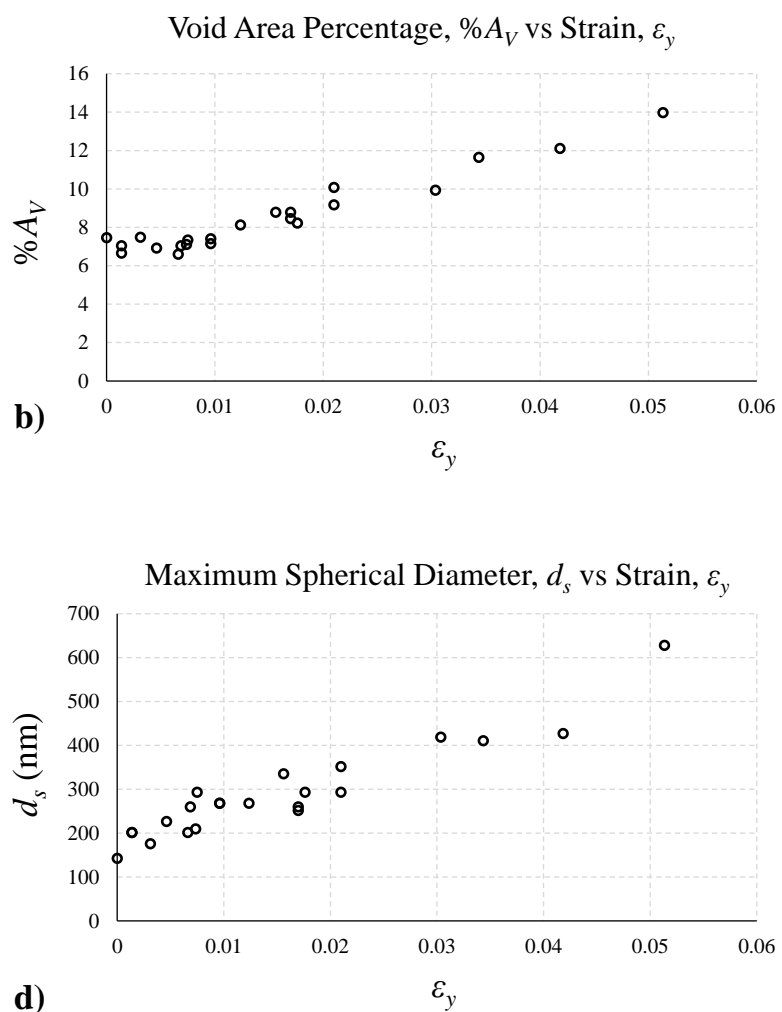

Figure 3: a) Pore parameters as measured from the central pore of the central repeating unit of the system and plots showing how the b) pore/void surface coverage, c) maximum pore dimension and d) maximum particle spherical diameter which may pass through the pore vary with increasing tensile strain.

As shown in Figure 3b, the void surface area of the system nearly doubles upon the application of $5 \%$ tensile strain, rising from nearly $7.5 \%$ up to $14 \%$. This large difference over such a small strain range is possible due to the quasi-closed configuration of the system which results in a very small initial porosity as well as the fact that the rotating squares mechanism is inherently characterised by large variation in pore dimensions upon loading [52]. Naturally, this increase in overall pore area is followed by a relatively small decrease in the maximum pore dimension, which shows a reduction of $c a$. $200 \mathrm{~nm}$ in magnitude from an initial length of $8 \mu \mathrm{m}$ (see Figure 3c). Given the high sensitivity of optical properties of nano-structured metamaterials to nanometric lattice parameters, the large changes in porosity and aperture shape observed in this system could make it ideal for use as a reconfigurable plasmonic metamaterial which may be potentially implemented as a UV, visible light or IR modulator. Another point of interest which is worth mentioning is the large change in the permissible maximum spherical diameter passable through the metamaterial pores. As shown in Figure 3d, the $d_{s}$ value rises from an initial value of $150 \mathrm{~nm}$ (equal to the slit thickness) up to a value of $620 \mathrm{~nm}$ at $5 \%$ strain. This 
fourfold increase, coupled with the alignment of the pore surface area with the $x y$-plane, indicates that this metamaterial system could be extremely well suited for use in minute-scale filtration applications, in particular as a mechanically-induced tuneable nano-filter for small particulate matter or microbes.

At this point, it is important to comment on the effect of FIB-milling damage on the metamaterial geometry. As reported in the pertinent literature $[19,45,53]$, the layer affected by the FIB can span from a few to several nanometres in terms of thickness. In particular, it has been experimentally observed and simulated that for very small grazing angles of the ion beam (e.g. $10^{\circ}$ ), the damaged layer is generally thinner than $30 \mathrm{~nm}[45,54]$. Although this dimension is rather limited, it is important to note that it is approximately one tenth of the in-plane separation distance $(300 \mathrm{~nm})$ and therefore, being $20 \%$ of the total joint thickness, may have an impact on the elastic behaviour of the entire metamaterial structure. Although, in this case, this effect does not appear to play a significant role in determining the deformation mechanism and Poisson's ratio of the system, it is expected to have a significant influence on the stiffness and strain tolerance of this metamaterial. A full investigation of this effect is beyond the scope of this study; however, it would be of great interest in the future to examine closely this aspect of FIB-milling on the mechanical performance of micro-/nano-architectured metamaterials.

Before concluding, it is worth highlighting the fact that the functionality of the planar metamaterial fabricated here is established primarily by smart design of the system. It is wellknown in the field of auxetic metamaterials, that the design of thin-sheet 2D mechanical metamaterials which deform primarily in-plane entails a considerable challenge even at the macroscale in view of the inherent propensity of thin systems to undergo out-of-plane deformations. In addition to this, the use of an ultra-thin membrane, the milling resolution constraints and the amplified effect of manufacturing residual stress factors at the nano-scale, present substantial hurdles to the design of the system which make the choice of base metamaterial geometry and lattice parameters an essential factor in ensuring that the desired functionality is achieved. The rotating square mechanism designed through the introduction of alternating straight-line slits is particularly ideal for this purpose. The geometry is relatively simple to mill, in terms of the required ion-beam pathway, hence reducing the number of possible defects, while it does not contain any ligaments, like other classic auxetic systems such as chiral and re-entrant honeycombs, which must be suitably thin (i.e. length to thickness ratio $\geq 10$ ) in order for the system to deform in an auxetic manner. Moreover, the system also has few degrees of freedom for in-plane deformation which makes it extremely resistant [55] 
to changes in deformation mechanism as a result of minor defects such as uneven thickness of the slit perforation or slight deviations from the proper positioning of the cut. Finally, provided that the length, $p$, of the perforation is very long in comparison to the other lattice parameters, the extent of out-of-plane deformation is determined by the separation parameter, $s$, which must be of smaller or comparable magnitude to the out-of-plane thickness of the system, $d$, to ensure in-plane dominated deformation. In this case, the $d / s$ ratio was 1.2 , which guarantees that the system undergoes mainly in-plane deformation at small strains, although the fact that this ratio is very small suggests that this might not remain the case in the event that significantly higher tensile loading is applied, provided that the system can tolerate such loads without fracturing. Another advantage of using this slit perforated geometry is that, besides the small initial porosity, milling/fabrication of the system takes considerably less time than it would take for a corresponding area perforated system due to the relatively small surface area which must be removed. Lastly, it is also important to mention that while the metamaterial was actuated insitu using a nanomanipulator in this work, this is not the only method through which microactuation may be induced. Recent advances in MEMS and NEMS research suggest that actuation could also be potentially electrically or thermally induced [12,34,56,57], while studies on macroscale magnetic mechanical metamaterials [58,59] could also provide a viable route towards the development of suitable micro-actuation devices.

The work conducted in this study could potentially be used as a blueprint for the design of twodimensional nano-porous reconfigurable metamaterials. Although the base design of the rotating square geometry was used in this study, previous works on macroscale auxetic systems $[46,50,51]$ have shown that a variety of slit perforation patterns exist which may be used to design planar nanostructures based on similar deformation modes. This could potentially open up the possibility of manufacturing nanostructured metamaterials with a wide range of aperture shapes and sizes which can be reconfigured through actuation, particularly if the slit patterning is based on a metamaterial with the potential to exhibit a large negative Poisson's ratio. Obviously, the out-of-plane thickness size limitations and material failure criteria highlighted previously for the design of this metamaterial would also apply for these systems and would need to be overcome in order to realise these geometries at such as small scale. However, this study has shown that if these factors are taken into account during the pre-design phase and the necessary precautions for correct loading of the metamaterial are employed, then the fabrication of similar metamaterials based on more complex and challenging geometries should not prove an unattainable objective. Such metamaterial geometries with highly tuneable pore 
shapes and sizes at such a small scale would be ideal for implementation as reconfigurable optical metamaterials, of which current designs found in literature have already shown promise for possible future use as electromagnetic cloaking devices [60]. In addition, the added versatility of these metamaterials could also be potentially advantageous for applications involving filtration of micro- or nanoscopic particles with a variety of shapes and sizes.

\section{Conclusion}

To summarise, in this work we have designed for the first time an ultrathin 2D auxetic metamaterial which deforms primarily in-plane using FIB-milling. We tested the system insitu by actuation stimulated through the use of a nanomanipulator and observed its deformation using SEM. The metamaterial exhibits a negative Poisson's ratio of -0.78 and the deformation of this system was analysed by comparison with corresponding FE simulations and experimental tests on macroscale thick and thin metamaterials made from rubber and Kapton respectively. An analysis of the porosity and aperture shape changes upon loading of the system showed that the porosity of the system nearly doubles over a 5\% tensile strain range, while the maximum permissible diameter of a spherical particle passing through the apertures increases fourfold, with ranges from $150 \mathrm{~nm}$ to $600 \mathrm{~nm}$. These properties make these systems ideal candidates for implementation as reconfigurable plasmonic metamaterials as well as for use in nanofiltration applications and also highlights the vast unexplored potential of replicating macro-scale 2D auxetic mechanisms at the micro- and nano-level.

\section{Acknowledgements}

L.M. and A.M.K. would like the thank the Royal Society for the Commonwealth Science Conference Follow-On Mentoring Grant 2017 CSC\R1\170018 which enabled this collaboration. The authors would like to thank Prof. Alfonso Castrejon-Pita for providing access to the laser cutter used to fabricate the macroscale metamaterials and Daniele Treacher for kindly providing the $\mathrm{Si}_{3} \mathrm{~N}_{4}$ base material.

\section{Data Availability Statement}


Certain raw data required to reproduce these findings cannot be shared at this time due to technical limitations. Additional data and information is also provided in the Supplementary Information.

\section{References}

[1] van Assenbergh P, Meinders E, Geraedts J, Dodou D. Nanostructure and Microstructure Fabrication : From Desired Properties to Suitable Processes. Small 2018;14:1703401. doi:10.1002/smll.201703401.

[2] Hohmann JK, Renner M, Waller EH, von Freymann G. Three-Dimensional u-Printing: An Enabling Technology. Adv Opt Mater 2015;3:1488-507.

[3] Iturri J, Xue L, Kappl M, García-fernández L, Barnes WJP. Torrent Frog-Inspired Adhesives : Attachment to Flooded Surfaces. Adv Funct Mater 2015;25:1499-505. doi:10.1002/adfm.201403751.

[4] Fozdar DY, Soman P, Lee JW, Han LH, Chen S. Three-dimensional polymer constructs exhibiting a tunable negative poisson's ratio. Adv Funct Mater 2011;21:2712-20. doi:10.1002/adfm.201002022.

[5] Bückmann T, Stenger N, Kadic M, Kaschke J, Frölich A, Kennerknecht T, et al. Tailored 3D Mechanical Metamaterials Made by Dip-in Direct-Laser-Writing Optical Lithography. Adv Mater 2012:2710-4. doi:10.1002/adma.201200584.

[6] Tricinci O, Terencio T, Mazzolai B, Pugno NM, Greco F, Mattoli V. 3D Micropatterned Surface Inspired by Salvinia molesta via Direct Laser Lithography. ACS Appl Mater Interfaces 2015;7:25560. doi:10.1021/acsami.5b07722.

[7] Park J, Hardy M, Kang SJUN, Barton K, Adair K, Mukhopadhyay DK, et al. Highresolution electrohydrodynamic jet printing. Nat Mater 2007;6:782-9. doi:10.1038/nmat1974.

[8] Uchida S, Ozaki N, Nakahama T, Oda H, Ikeda N, Sugimoto Y. Ultra-small nearinfrared multi-wavelength light source using a heterojunction photonic crystal waveguide and self-assembled InAs quantum dots. Jpn J Appl Phys 2017;56:050303.

[9] Gardner DF, Evans JS, Smalyukh II. Molecular Crystals and Liquid Crystals Towards 
Reconfigurable Optical Metamaterials : Colloidal Nanoparticle Self-Assembly and Self-Alignment in Liquid Crystals. Mol Cryst Liq Cryst 2011;545:1227-45. doi:10.1080/15421406.2011.571966.

[10] Ansari K, Kan JA Van, Bettiol AA, Watt F. Fabrication of high aspect ratio $100 \mathrm{~nm}$ metallic stamps for nanoimprint lithography using proton beam writing. Appl Phys Lett 2014;476:17-20. doi:10.1063/1.1773933.

[11] Jeong JW, Yang SR, Hur YH, Kim SW, Baek KM, Yim S, et al. High-resolution nanotransfer printing applicable to diverse surfaces via interface-targeted adhesion switching. Nat Commun 2014;5:5387. doi:10.1038/ncomms6387.

[12] Zheludev NI, Plum E. Reconfigurable nanomechanical photonic metamaterials. Nat Nanotechnol 2016;11:16-22. doi:10.1038/nnano.2015.302.

[13] Stewart ME, Anderton CR, Thompson LB, Maria J, Gray SK, Rogers JA, et al. Nanostructured Plasmonic Sensors. Chem Rev 2008;108:494-521.

[14] Choi D, Lee KY, Lee KH, Kim ES, Kim TS, Lee SY, et al. Piezoelectric touchsensitive flexible hybrid energy harvesting nanoarchitectures. Nanotechnology 2010;21:405503. doi:10.1088/0957-4484/21/40/405503.

[15] Tripathy A, Sen P, Su B, Briscoe WH. Natural and bioinspired nanostructured bactericidal surfaces. Adv Colloid Interface Sci 2017;248:85-104. doi:10.1016/j.cis.2017.07.030.

[16] Krogmeier JR, Dunn RC. Focused ion beam modification of atomic force microscopy tips for near-field scanning optical microscopy. Appl Phys Lett 2001;79:4494. doi:10.1063/1.1430028.

[17] Kim C, Ahn S, Jang D. Review : Developments in micro / nanoscale fabrication by focused ion beams. Vaccum 2012;86:1014-35. doi:10.1016/j.vacuum.2011.11.004.

[18] Machalett F, Seidel P. Focused Ion Beams and Some Selected Applications. Encycl. Appl. Phys., 2019, p. 1-36.

[19] Salvati E, Sui T, Lunt AJG, Korsunsky AM. The effect of eigenstrain induced by ion beam damage on the apparent strain relief in FIB-DIC residual stress evaluation. Mater Des 2016;92:649-58. doi:10.1016/j.matdes.2015.12.015. 
[20] Brostow W, Gorman BP, Olea-mejia O. Focused ion beam milling and scanning electron microscopy characterization of polymer + metal hybrids. Mater Lett 2007;61:1333-6. doi:10.1016/j.matlet.2006.07.026.

[21] Moll PJW. Focused Ion Beam Microstructuring of Quantum Matter. Annu Rev Condens Matter Phys 2018;13:147-62.

[22] Yao K, Liu Y. Plasmonic metamaterials. Nanotechnol Rev 2014;3:177-210. doi:10.1515/ntrev-2012-0071.

[23] Biener BJ, Nyce GW, Hodge AM, Biener MM, Hamza A V, Maier SA. Nanoporous Plasmonic Metamaterials. Adv Mater 2008;20:1211-7. doi:10.1002/adma.200701899.

[24] Kollmann H, Piao X, Esmann M, Becker SF, Hou D, Huynh C, et al. Toward Plasmonics with Nanometer Precision: Nonlinear Optics of Helium-Ion Milled Gold Nanoantennas. Nano Lett 2014:4778-84.

[25] Enkrich BC, Pérez-willard F, Gerthsen D, Zhou J, Koschny T, Soukoulis CM, et al. Focused-Ion-Beam Nanofabrication of Near-Infrared Magnetic Metamaterials. Adv Mater 2005;17:2547-9. doi:10.1002/adma.200500804.

[26] Casse BDF, Moser HO, Jian LK, Bahou M, Wilhelmi O, Saw BT, et al. Fabrication of 2D and 3D Electromagnetic Metamaterials for the Terahertz Range. J Phys Conf Ser 2006;34:885-90. doi:10.1088/1742-6596/34/1/147.

[27] Huang W, Zhang Y, Tang X, Cai L, Zhao J, Zhou L. Optical properties of a planar metamaterial with chiral symmetry breaking. Opt Lett 2011;36:3359-61.

[28] Esposito M, Tasco V, Todisco F, Benedetti A, Sanvitto D, Passaseo A. Three Dimensional Chiral Metamaterial Nanospirals in the Visible Range by Vertically Compensated Focused Ion Beam. Adv Opt Mater 2014;2:154-61. doi:10.1002/adom.201300323.

[29] Chen Y, Gao J, Yang X. Chiral Metamaterials of Plasmonic Slanted Nanoapertures with Symmetry Breaking. Nano Lett 2018;18:520-7. doi:10.1021/acs.nanolett.7b04515.

[30] Hentschel M, Schäferling M, Duan X, Giessen H, Liu N. Chiral plasmonics. Sci Adv 2017;3:1602735. 
[31] Liu Z, Du H, Li J, Lu L, Li Z, Fang NX. Nano-kirigami with giant optical chirality. Sci Adv 2018;4:1-9.

[32] Pitchappa P, Ho CP, Cong L, Singh R, Singh N, Lee C. Reconfi gurable Digital Metamaterial for Dynamic Switching of Terahertz Anisotropy. Adv Opt Mater 2016;4:391-8. doi:10.1002/adom.201500588.

[33] Valente J, Plum E, Youngs IJ, Zheludev NI. Nano- and Micro-Auxetic Plasmonic Materials. Adv Mater 2016:5176-80. doi:10.1002/adma.201600088.

[34] Gholipour B, Piccinotti D, Karvounis A, Macdonald KF, Zheludev NI. Reconfigurable Ultraviolet and High-Energy Visible Dielectric Metamaterials. Nano Lett 2019;19:1643-8. doi:10.1021/acs.nanolett.8b04576.

[35] Evans, K.E., Nkansah, M.A., Hutchinson, J., Rogers SC. Molecular Network Design. Nature 1991;353:124.

[36] Lakes R. Foam Structures with a Negative Poisson's Ratio. Science (80- ) 1987;235:1038-40. doi:10.1126/science.235.4792.1038.

[37] Wojciechowski KW, Branka AC. Negative Poisson ratio in a two-dimensional “"isotropic"” solid. Phys Rev A 1989;40:7222-5.

[38] Prall D, Lakes RS. Properties of a chiral honeycomb with a Poisson's ratio of -1. Int J Mech Sci 1997;39:305-14.

[39] Alderson A, Alderson KL, Attard D, Evans KE, Gatt R, Grima JN, et al. Elastic constants of 3-, 4- and 6-connected chiral and anti-chiral honeycombs subject to uniaxial in-plane loading. Compos Sci Technol 2010;70:1042-8. doi:10.1016/j.compscitech.2009.07.009.

[40] Grima JN, Evans KE. Auxetic behavior from rotating squares. J Mater Sci Lett 2000;19:1563-5.

[41] Grima JN, Evans KE. Auxetic behavior from rotating triangles. J Mater Sci 2006;1:3193-6. doi:10.1007/s10853-006-6339-8.

[42] Masters IG, Evans KE. Models for the elastic deformation of honeycombs. Compos Struct 1996;35:403-22.

[43] Gibson LJ, Ashby MF, Schajer GS, Robertson CI. The Mechanics of Two 
Dimensional Cellular Materials. Proc R Soc A Math Phys Eng Sci 1982;382:25-42.

[44] Rothenburg L, Berlin AA, Bathurst RJ. Microstructure of isotropic materials with a negative Poisson's ratio. Nature 1991;354:470-2.

[45] Salvati E, Brandt LR, Papadaki C, Zhang H, Mousavi SM, Wermeille D, et al. Nanoscale structural damage due to focused ion beam milling of silicon with Ga ions. Mater Lett 2018;213:346-9. doi:10.1016/j.matlet.2017.11.043.

[46] Mizzi L, Azzopardi KM, Attard D, Grima JN, Gatt R. Auxetic metamaterials exhibiting giant negative Poisson's ratios. Phys Status Solidi - Rapid Res Lett 2015;9:425-30. doi:10.1002/pssr.201510178.

[47] Shan S, Kang SH, Zhao Z, Fang L, Bertoldi K. Design of planar isotropic negative Poisson's ratio structures. Extrem Mech Lett 2015;4:96-102. doi:10.1016/j.eml.2015.05.002.

[48] Cho Y, Shin J, Costa A, Ann T, Kunin V, Li J, et al. Engineering the shape and structure of materials by fractal cut. Proc Natl Acad Sci 2014;111:17390-5. doi:10.1073/pnas.1417276111.

[49] On the origin of auxetic behaviour in the silicate $\alpha$-cristobalite - Grima, Gatt, Alderson, Evans.pdf n.d.

[50] Grima JN, Gatt R. Perforated Sheets Exhibiting Negative Poisson's Ratios. Adv Eng Mater 2010;12:460-4. doi:10.1002/adem.201000005.

[51] Mizzi L, Salvati E, Spaggiari A, Tan J, Korsunsky AM. Highly stretchable twodimensional auxetic metamaterial sheets fabricated via direct-laser cutting. Int J Mech Sci 2020;167:105242. doi:10.1016/j.ijmecsci.2019.105242.

[52] Attard D, Casha AR, Grima JN. Filtration Properties of Auxetics with Rotating Rigid Units. Materials (Basel) 2018;11:1-13. doi:10.3390/ma11050725.

[53] Wang YC, Xie DG, Ning XH, Shan ZW. Thermal treatment-induced ductile-to-brittle transition of submicron-sized Si pillars fabricated by focused ion beam. Appl Phys Lett 2015;106:081905.

[54] Guenole J, Prakash A, Bitzek E. Atomistic simulations of focused ion beam machining of strained silicon. Appl Surf Sci 2017;416:86-95. 
[55] Grima JN, Mizzi L, Azzopardi KM, Gatt R. Auxetic Perforated Mechanical Metamaterials with Randomly Oriented Cuts. Adv Mater 2016;28:385-9. doi:10.1002/adma.201503653.

[56] Karvounis A, Aspiotis N, Zeimpekis I, Ou J, Huang C, Hewak D, et al. Mechanochromic Reconfigurable Metasurfaces. Adv Sci 2019;6:1900974. doi:10.1002/advs.201900974.

[57] Ou J, Plum E, Zhang J, Zheludev NI. An electromechanically reconfigurable plasmonic metamaterial operating in the near-infrared. Nat Nanotechnol 2013:1-4. doi:10.1038/nnano.2013.25.

[58] Dudek KK, Gatt R, Grima JN. 3D composite metamaterial with magnetic inclusions exhibiting negative stiffness and auxetic behaviour. Mater Des 2020;187:108403. doi:10.1016/j.matdes.2019.108403.

[59] Slesarenko V. Planar Mechanical Metamaterials with Embedded Permanent Magnets. Materials (Basel) 2020;13:1-12.

[60] Shin D, Urzhumov Y, Jung Y, Kang G, Baek S, Choi M, et al. Broadband electromagnetic cloaking with smart metamaterials. Nat Commun 2012;3:1213. 